\title{
On the sum and the difference of finite sets of integers
}

\section{Reinhard A. Razen}

Let $A=\left\{a_{i}\right\}$ be a finite set of integers and let $p$ and $m$ denote the cardinalities of $A+A=\left\{a_{i}+a_{j}\right\}$ and $A-A=\left\{a_{i}-a_{j}\right\}$, respectively. In the paper relations are established between $p$ and $m$; in particular, if $\max _{i}\left(a_{i}-a_{i-1}\right)=2$ those sets are characterized for which $p=m$ holds.

Let $A=\left\{a_{i} \mid 0 \leq i \leq n\right\}$ be a finite set of integers where without loss of generality we may assume $a_{0}<a_{1}<\ldots<a_{n}$, and let $p$ and $m$ denote the cardinalities of $A+A=\left\{a_{i}+a_{j} \mid a_{i}, a_{j} \in A\right\}$ and $A-A=\left\{a_{i}-a_{j} \mid a_{i}, a_{j} \in A\right\}$, respectively. Spohn [2] remarked that the values of $p$ and $m$ depend only on the $n$ differences $d_{i}=a_{i}-a_{i-1}$ $(1 \leq i \leq n)$ and are unchanged if the $d_{i}$ are multiplied by a constant or are reversed. Thus we may set $a_{0}=0$ and $\left(a_{1}, a_{2}, \ldots, a_{n}\right)=1$.

Further we use the abbreviation $\max _{1 \leq i \leq n} d_{i}=D$.

Macdonald and Street [1] proved the following.

THEOREM. If $D \leq 2$, then $p \leq m$.

The proof is based on the following results.

Received 28 April 1976. 
LEMMA A. If $d_{1}=1$, then

$$
A-A=\left\{k \in Z \mid-a_{n} \leq k \leq a_{n}\right\} \text {. }
$$

LEMMA B. If

$$
d_{1}=\ldots=d_{\alpha}=d_{n-\beta+1}=\ldots=d_{n}=2, d_{\alpha+1}=d_{n-\beta}=1,
$$

then

$$
m=2 a_{n}+1-2 \min (\alpha, \beta)
$$

and

$$
p \leq 2 a_{n}+1-(\alpha+\beta)
$$

REMARK. In Lemma $B$ the value of $p$ can be improved to $p=2 a_{n}+1-(\alpha+\beta)$, since if

$$
A=\left\{0,2,4, \ldots, 2 \alpha, 2 \alpha+1, \ldots, a_{n}-2 \beta-1, a_{n}-2 \beta, \ldots, a_{n}-2, a_{n}\right\} \text {, }
$$

we have

$A+A=\left\{0,2,4, \ldots, 2 \alpha, 2 \alpha+1, \ldots, 2 a_{n}-2 \beta-1,2 a_{n}-2 \beta, \ldots, 2 a_{n}-2,2 a_{n}\right\}$, where all integers between $2 \alpha$ and $2 \alpha_{n}-2 \beta$ belong to $A+A$, but for numbers below $2 \alpha$ and beyond $2 a_{n}-2 \beta$ this holds only for even integers.

Using these results we shall derive the following facts.

LEMMA 1. If $d_{1}=\ldots=d_{D-1}=1$, then

$$
A-A=\left\{k \in Z \mid-a_{n} \leq k \leq a_{n}\right\} \text {. }
$$

Proot. The proof is similar to that of Lemma A in [1], namely, by induction on $k$ it is shown that each $k \in\left\{1, \ldots, a_{n}\right\}$ is the sum of consecutive $d_{i}$ 's, that is $k=d_{s}+d_{s+1}+\ldots+d_{t}$, where $s \in\{1, \ldots, D\}$ and $t \geq s$.

(1) $k=1: k=d_{1}$.

(2) Suppose the claim is true for $k-1=d_{s}+\ldots+d_{t}$. If $s>1$, then 


$$
k=d_{s-1}+d_{s}+\ldots+d_{t} .
$$

If $s=1$ and $d_{t+1}=1$, then

$$
k=d_{s}+\ldots+d_{t}+d_{t+1} .
$$

If $s=1$ and $d_{t+1}>1$, then

$$
k=d_{d_{t+1}}+d_{d_{t+1}+1}+\ldots+d_{t+1}
$$

since, on account of $d_{t+1} \leq D$, we have $d_{1}=\ldots=d_{d_{t+1}-1}=1$.

Therefore we subtracted $d_{t+1}-1$ from $k-1$ and then we added $d_{t+1}$.

THEOREM 1. If

$$
d_{1}=\ldots=d_{D-1}=d_{n-D+2}=\ldots=d_{n}=1 \text {, }
$$

then

$$
\begin{aligned}
& \text { (a) } A+A=\left\{k \in Z \mid 0 \leq k \leq 2 a_{n}\right\} \text {, and } \\
& \text { (b) } p=m
\end{aligned}
$$

hold.

Proot. We show claim (a) in three stages.

1. Let $0 \leq k \leq a_{n}-1$. If $k \in A$, then, on account of $0 \in A$, we have $k=k+0 \in A+A$. If $k \notin A$, then at least one of the integers between $k-D+1$ and $k-1$ is an element of $A$; otherwise $D$ consecutive integers would not belong to $A$ which contradicts the definition of $D$. Now if $k-j \in A \quad(j \in\{1,2, \ldots, D-1\})$, we have, since $j \in A$, also $k=(k-j)+j \in A+A$.

2. If $k=a_{n}$, then $k=k+0 \in A+A$.

3. Let $a_{n}+1 \leq k \leq 2 a_{n}$. If $k=a_{n}+a_{j}$ for one $j \in\{1, \ldots, n\}$, then $k \in A+A$. Now let us assume that $k \neq a_{n}+a_{j}$ for all $j \in\{1,2, \ldots, n\}$. Then for a suitable $j_{1} \in\{1, \ldots, n-1\}$ we have 


$$
a_{n}+a_{j_{1}}<k<a_{n}+a_{j_{1}+1}
$$

From this we derive $2 \leq d_{j_{1}+1}=a_{j_{1}+1}-a_{j_{1}} \leq D$ and with a suitable $b \in\left\{1, \ldots, d_{j_{1}+1}-i\right\}$ we have

$$
k=a_{n}+a_{j_{1}}+b=a_{n}+a_{j_{1}+1}-a_{j_{1}+1}+b=\left(a_{n}-a_{j_{1}+1}+b\right)+a_{j_{1}+1} .
$$

On account of

$$
a_{n}-d_{j_{1}+1}+b \geq a_{n}-d_{j_{1}+1}+1 \geq a_{n}-D+1
$$

$a_{n}-d_{j_{1}+1}+b$ belongs to $A$, and therefore $k \in A+A$.

Claim (b) follows immediately from (a) together with Lemma 1 . The example $A=\{0,1,2,3,4,9,10,12,13\}$ with $A+A=\{0,1, \ldots, 26\}$ and $A-A=\{0, \pm 1, \ldots, \pm 13\}$ shows that the converse of Theorem 1 does not hold. But we are able to show the following weaker statement.

LEMMA 2. Let $p=m$ and $d_{1}=\ldots=d_{D-1}=1$. Then $d_{n}=1$ and $d_{n-1} \in\{1,2\}$ hold.

Proof. By Lemma 1 we have $m=2 a_{n}+1$. By hypothesis $p=m$, therefore $A+A$ must consist of all integers between 0 and $2 a_{n}$. If $d_{n}>1$, then $2 a_{n}-1$ is not representable as the sum of two elements of $A$; the same holds for $2 a_{n}-3$, if $d_{n-1}>2$.

After these preliminaries we are able to characterize those sets with $D=2$, for which $p=m$ holds. For this purpose let $\alpha$, respectively $\beta$, denote the number of differences at the ends of the set $A$ which are equal to 2 . $\left(\alpha=0\right.$ means $d_{1}=1 ; \alpha>0$ means $d_{1}=\ldots=d_{\alpha}=2$, $\left.d_{\alpha+1}=1.\right)$

THEOREM 2. Let $A$ be a set of integers with $D=2$. Then $p=m$ if and only if $\alpha=B$.

proot. First we prove the theorem for $\alpha=0$, that is, $d_{1}=1$. If $B=0$, then by Theorem $I(b)$, we have $p=m$. If conversely $p=m$, we 
obtain by Lemma 2 that $d_{n}=1$, that is, $\alpha=\beta=0$.

Now let $\alpha>0$. The necessity follows from Lemma $B$, since for $\alpha \neq \beta$ the inequality $p<m$ holds. Finally, the sufficiency is a consequence of our remark to Lemma $\mathrm{B}$, which states that $p=m=2 a_{n}+1-2 \alpha$.

Now we shall investigate which values can be assumed by $p=m$.

THEOREM 3. (a) Let $A$ be a set with $n+1$ elements $(n \geq 2)$, $D \leq 2$, and $p=m$. Then $p$ is an odd integer from the interval $2 n+1 \leq p \leq 4 n-3$.

(b) Conversely, to each such $p$ there exists a set $A$ with $n+1$ elements and $D \leq 2$, for which $|A+A|=|A-A|=p$.

Proot. (a) If $\alpha=\beta=0$, that is $d_{1}=d_{n}=1$, for the largest element of $A$ the inequalities $n \leq a_{n} \leq 2(n-2)+2=2 n-2$ hold, therefore using Theorem 1 (a) we obtain $2 n+1 \leq p \leq 4 n-3$.

If, on the other hand $\alpha=\beta>0$, we have $n+2 \alpha \leq a_{n} \leq 2 n-2$ for even $n$ and $n+2 \alpha \leq a_{n} \leq 2 n-1$ for odd $n$. Then

$$
2 n+1<2 n+2 \alpha+1 \leq p \leq 3 n-1 \leq 4 n-3 \text { for even } n \geq 2
$$

from our remark to Lemma $B$, and also

$$
2 n+1<2 n+2 \alpha+1 \leq p \leq 3 n \leq 4 n-3 \text { for odd } n \geq 3 \text {. }
$$

We prove (b) showing by induction on $n$ that each possible value of $p$ can be generated by a set with $d_{1}=d_{n}=1$. For $n=2$ the set $A=\{0,1,2\}$ satisfies our theorem. Now let $p \in\{2 n-1,2 n+1, \ldots, 4 n-7\}$ and be arbitrary. By hypothesis there exists a set $A$ with $n$ elements and $d_{1}=d_{n-1}=1$, for which $|A+A|=p$ holds.

If we form $A_{1}=A \cup\left\{a_{n-1}+1\right\}$, we have

$$
A_{1}+A_{1}=(A+A) \cup\left\{2 a_{n-1}+1,2 a_{n-1}+2\right\} \text {, }
$$

therefore $\left|A_{1}+A_{1}\right|=p+2$. Finally, by Theorem 1 (a) the set $A_{2}$ with $d_{1}=d_{n}=1$ and $d_{2}=\ldots=d_{n-1}=2$ has 


$$
\left|A_{2}+A_{2}\right|=2 a_{n}+1=2(2 n-2)+1=4 n-3 .
$$

THEOREM 4. AlZ values for $p=m$ which are possible according to Theorem 3 can be generated by symmetric sets (that is $d_{i}=d_{n-i+1}$ ), whilst asymetric sets generate all values for $p=m$ except the smallest $(2 n+1)$ and the largest (4n-3).

Proof. First we prove the claim for symmetric sets and even $n$ and we consider sets with the following difference schemes:

$$
\begin{aligned}
& \text { (a) } d_{1}=\ldots=d_{n}=1 ; \\
& \text { (b) } d_{1}=\ldots=d_{(n-2 j) / 2}=d_{(n+2 j+2) / 2}=\ldots=d_{n}=1, \\
& d_{(n-2 j+2) / 2}=\ldots=d_{(n+2 j) / 2}=2 \text { for } 1 \leq j \leq(n-2) / 2 ; \\
& \text { (c) } d_{1}=d_{(n-2 j+2) / 2}=\ldots=d_{(n+2 j) / 2}=d_{n}=2, \\
& d_{2}=\ldots=d_{(n-2 j) / 2}=d_{(n+2 j+2) / 2}=\ldots=d_{n-1}=1 \text { for } \\
& 0 \leq j \leq(n-4) / 2 .
\end{aligned}
$$

By Theorem $I(a)$ and the remark to Lemma $B$ for the corresponding values of $p=m$ we obtain:

(a) $p=2 n+1$;

(b) $p=2 a_{n}+1=2 n+4 j+1$; on account of the possible values for $j$ follows $p \in\{2 n+5,2 n+9, \ldots, 4 n-7,4 n-3\}$;

(c) $p=2 a_{n}+1-2=2 n+4 j+3$, therefore $p \in\{2 n+3,2 n+7, \ldots, 4 n-9,4 n-5\}$.

Now let $n$ be odd. We consider the differences:

(a) $d_{1}=\ldots=d_{n}=1$;

(b) $d_{1}=\ldots=d_{(n-2 j-1) / 2}=d_{(n+2 j+3) / 2}=\ldots=d_{n}=1$, $d_{(n-2 j+1) / 2}=\ldots=d_{(n+2 j+1) / 2}=2$ for $0 \leq j \leq(n-3) / 2$;

(c) $d_{1}=d_{(n-2 j+1) / 2}=\ldots=d_{(n+2 j+1) / 2}=d_{n}=2$, $d_{2}=\ldots=d_{(n-2 j-1) / 2}=d_{(n+2 j+3) / 2}=\ldots=d_{n-1}=1$ for 


$$
0 \leq j \leq(n-5) / 2 \text {. }
$$

In these cases for the values of $p=m$ we obtain:

(a) $p=2 n+1$;

(b) $p=2 a_{n}+1=2 n+4 j+3$, therefore $p \in\{2 n+3,2 n+7, \ldots, 4 n-7,4 n-3\} ;$

(c) $p=2 a_{n}+1-2=2 n+4 j+5$, therefore $p \in\{2 n+5,2 n+9, \ldots, 4 n-9,4 n-5\}$.

If we form for $n \geq 4$ those asymmetric sets $A$ which are defined by $d_{1}=\ldots=d_{j}=d_{n}=1, d_{j+1}=\ldots=d_{n-1}=2(2 \leq j \leq n-2)$, then by Theorem 1 (a) for $|A+A|$ we obtain the values $4 n-2 j-1$; therefore $p \in\{2 n+3,2 n+5, \ldots, 4 n-7,4 n-5\}$.

Now let $A$ be an asymmetric set with $p=m$. If $d_{1}=d_{n}=1$, the inequalities $2 n+3 \leq|A+A| \leq 4 n-5$ hold. If

$$
d_{1}=\ldots=d_{\alpha}=d_{n-\alpha+1}=\ldots=d_{n}=2 \text {, }
$$

$d_{\alpha+1}=d_{n-\alpha}=1$, then, on account of the asymmetry, we have

$\alpha+1 \neq n-\alpha$; therefore $n+2 \alpha+1 \leq a_{n} \leq 2 n-3$. Since $\alpha \geq 1$, for $p=2 a_{n}+1-2 \alpha$ we obtain the inequalities

$$
2 n+5 \leq 2 n+2 \alpha+3 \leq p \leq 4 n-2 \alpha-5 \leq 4 n-7,
$$

which prove Theorem 4 .

\section{References}

[1] Sheila Oates Macdonald and Anne Penfold Street, "On Conway's conjecture for integer sets", Bull. Austral. Math. Soc. 8 (1973), 355-358.

[2] William G. Spohn, Jr., "On Conway's conjecture for integer sets", Canad. Math. Bulz. 14 (1971), 461-462.

Institut für Mathematik und Mathematische Statistik, Montanuniversität Leoben, Leoben, Austria. 